\title{
The Lattice Boltzmann method and computational analysis of bone dynamics-I
}

\author{
Sana Javed ${ }^{1}$, Ayesha Sohail ${ }^{1}$, Khadija Maqbool ${ }^{2}$, Saad Ihsan Butt ${ }^{1}$ and Qasim Ali Chaudhry ${ }^{3^{*}}$
}

${ }^{*}$ Correspondence:

chqasim@uet.edu.pk

${ }^{3}$ Department

of Mathematics,

University of Engineering

and Technology,

Lahore 54890, Pakistan

Full list of author information

is available at the end of the article

\begin{abstract}
Bone is comprised of an enormously hierarchical construction that promotes transportation of necessary fluids and solids, guaranteeing accurate function and growth. Bone remodeling is a combined process of bone creation and destruction. A number of mathematical models have been developed for the balanced and imbalanced bone remodeling. A brief overview regarding mathematical modeling of bone remodeling is provided. The Lattice Boltzmann method (LBM) has widely been implemented in CFD simulations, and it is becoming more suitable in the application of image processing amongst several others. Mainly, the LBM simulates the communication between synthetic particles dispersed in a lattice. Canaliculi and tortuous channels that have more or less roughly circular structure link among oval bodies identified as lacunae, and are vital to the function of bone. As there is a lack of equipment to inspect flow in channels on the order of measure of canaliculi, so the use of computational methods are more advantageous to give perceptivities into the nature of the flows. In this article, the computational fluid dynamics analysis is descried, using the Lattice Boltzmann method, to examine the result of the microscopic surface roughness of the canalicular wall, which is formed by collagen fibrils, on the flow profiles in the pericellular space.
\end{abstract}

Keywords: Bone remodelling, Numerical analysis, In-silico techniques, Lattice Boltzmann method

\section{Introduction}

Organic tissues are composed of the cells and the extracellular milieu. The external environmental stimuli may induce several cellular activities inside the tissue which in turn can vary tissue matrix. Such stimuli can be mechanical, hormonal, chemical or electrical etc. In the case of mechanical stimuli, the cells within the subjected tissue undergo a few aspects of the mechanical stimulus, and decide to alter the tissue matrix in a pertinent manner depending upon the aptness of stimulus. The vertebrate skeleton plays a vital part in supplying mechanical support. Bone is a mineralized tissue and such mineralization is compulsory for its composition. Bone mineralization consists of a well organized practice of producing calcium phosphate by bone forming cells and put down in specific quantities inside the stringy matrix of bone. Bone is normally comprised of elastic collagen fiber besieged by firm hydroxyapatite mineral. A skeletal system is structurally strengthened by the noteworthy contribution of two main classes of bone tissue, called cancellous (trabecular or spongy) and cortical bone (haversian or compact). Cortical 
bone is a hard and dense tissue on the outer side of the femur with microscopically small channels and no marrow. Cancellous bone is a porous composition comprising of a system of stiff interrelated strands called trabeculae intermingled with marrow and a large number of small blood vessels. It lies in the inner side of the femur.

Mathematical modeling provides a powerful tool to reduce ambiguity, and allows to test various experimental and theoretical hypothesis that may be difficult or impossible to test in-vitro or in-vivo. There have been relatively few mathematical models proposed that study the integrated effects of known bonecell interactions.

In the field of mathematical biology, numerical schemes are usually based on the discretization of continuum equations. Such equations describe the biological problems at the macroscopic scale. The Lattice Boltzmann method, on the other hand has the feasibility to study the multi-scales models. Such models are more realistic and satisfy the current challenges of optimization, theory and research. For example, LBM is used in the field of oncology (Alemani et al. 2012), it can swiftly demonstrate the complex fluid flows of biological problems (Aidun and Clausen 2010), furthermore, the technique has been successfully used to model the flow through the bone tissues at the pore scale (Zeiser et al. 2008).

The micro-scopic models and the meso-scopic kinetic equations are usually involved in the multi-scale modelling. A two step procedure is involved, which reduces the complexity of the problem. First, the kinetic models are used to incorporate the physical properties of the process(es), then the averaged properties obtained, obey the desired macro-scopic equations (Mohamad 2011).

In this article we have described the importance of lattice Boltzmann method in the field of osteology. In "Bone" section, we have explained in simple words, the bone structure and dynamics. Next, in "Bone surface" section we have outlined the in-silico attempts to model bone dynamics. In "Bone dynamics and the Lattice Boltzmann method" section we have described the lattice Boltzman method and its applications in the field of osteology. In "Mathematical description of the Lattice Boltzman method" section, we have explained the method in detail. Finally, some important conclusions are drawn and future work is outlined.

\section{Bone}

At microscopic level, the principal unit of bone is osteon. In cortical bone, a typical osteon is a cylinder about 200 or $250 \mu \mathrm{m}$ in diameter and 1-2 cm in length. In trabecular bone, the osteons are the trabeculae and are ordered in a form beside the lines of average principle stress. Long axis of the cortical bone and all osteons, occur in parallel positions. Each osteon has a fluid occupied excavating or lumen along the center of its long axis. Every lumen contains a blood vessel which nourishes the bone cells in the locality of that osteon. A system interconnecting all these blood vessels and lumina is known as Haversian system. Twenty to thirty concentric lamellae together compose the wall of the osteon, the thickness of each lamella is about 70-100 $\mu \mathrm{m}$. A cement line besieging the surface of each osteon is a 1-2 $\mu \mathrm{m}$ dense layer of mineralized matrix lacking in collagen fibers. 
Compact bone adheres one of the three forms, namely woven, laminar and haversian bone, histologically. Woven bone is an unsystematic stringy bone, and has lesser mineral content comparatively to other two. With ontogenesis, the woven bone is transformed to laminar bone and at adulthood, partly to haversian bone. The geometric configuration of Osteon of haversian bone and the laminae of laminar bone of the same material are distinct. Bone cells basically lay in lacunae, which is an arrangement of cavities contained in a mutual border connecting laminae in both haversian and laminar bone and from which expand several fine canals called canaliculi.

\section{Bone surface}

Emergence of bone is confined to appositional growth for the reason that mineralized bone tissue is inflexible, due to which all processes of bone take place at the surface of the bone. Endosteal surface splits into the haversian, endocortical, and trabecular surfaces. More than $61 \%$ part of the bone surface comprises of trabecular surface (Hall et al. 2017). Resorbing, forming and resting are three operational states of bone surfaces, and at any particular instant bone undergoes one of these states. Osteoblasts and osteoid differentiate the bone forming surfaces while osteoclasts lie at the bone resorbing surfaces; quiescent or resting surface is liberated of both osteoclasts and osteoblasts. Bone lining cells lineup the resting surface. Quiescent is the most occurring state of the bone surfaces in the adults. Bone milieu and bone marrow get detached by the lining cells at a concentration a bit higher than its solvability.

\section{Cells of bone}

Mainly there are four categories of bone cells called osteoclasts, osteoblasts, osteocytes and bone lining cells. Osteoclasts are multinucleated cells carrying nuclei from 1 to more than 50. The major role of osteoclasts is bone resorption. Generally, resorption cavities on the bone surface contain active osteoclasts. Osteoblasts are the bone making cells and fabricate all components of the bone. The inner wall of the osteon, during its growth, gets narrower because of the embedding of the series of lamellar bone. Osteoblasts are the key factors of such implantation. Lamellar bone is a complex which is composed of a material like fiberglass. Bone formation consists of firstly the embedding of an organic milieu and then the mineralization of the milieu with salts from ions (mainly calcium and phosphate) in the extracellular fluid. Osteocytes are the mechano sensors and the most profuse cell type in adult bone (Mullender and Huiskes 1997). After the process of bone formation, in a fresh molded osteoid some old embedded osteoblasts get differentiated into osteocytes by dropping a lot of their organelles. Bone lining cell are the compressed and elongated inactive osteoblasts and cover the resting bone surface. Bone lining cells have the capability to form bone, following no former bone resorption, in reaction to bone anabolic agents and may govern mineral homeostasis with the composite of osteoblasts and osteocytes (Kerschnitzki et al. 2013).

\section{Bone remodeling}

The combined practice of bone resorption and regeneration is called bone remodeling. Due to mechanical stress of the skeleton, bones suffer local microdamages; bone remodeling allows the reinstatement of such microdamages. According to low or high 
mechanical strains of the skeleton bunches of bones are removed or created, respectively. Coupling is referred as the common remodeling process in which bone loss and gain remain balanced (Parfitt 1987). A chronic disturbance in this well structured system leads to distinct metabolic bone disorders like osteoporosis, cancer metastases and Pagets disease. A large reservoir of uncommitted mesenchymal progenitors provides an immense supply of osteoblasts lineages which differentiate further into osteoblast cells under the complex effects of specific factors like PTH and TGF- $\beta$ (Canalis et al. 1988). After bone formation, osteoblasts either expire or convert to either lining cells or osteocytes. RANK (A receptor activator nuclear factor kappa beta NF-) and RANKL (RANK Ligand) accelerate the differentiation of osteoclastic progenitors into more mature osteoclasts. Osteoblasts express RANKL on its exterior which attaches to RANK located on the surface of precursors of osteoclasts, thus promoting the differentiation and activation of osteoclasts. After bone resorption osteoclasts die. Stromal/osteoblastic cells also generate a soluble decoy receptor called osteoprotegerin (OPG) which pessimistically control the contact of RANK and RANKL. RANKL is captured by OPG receptor which in turn inhibits the stimulation of RANK expressed by osteoclast. The basic regulatory system to control bone remodeling is based on this RANK-RANKL-OPG signaling pathway. The molecular and cellular communications generated through such complex provide the basic dynamics accountable for sustaining the firm coupling among osteoblasts and osteoclasts for the period of ordinary bone turnover.

\section{Fluid in "bone"}

The study that deals with modeling the connection between deformation and fluid flow in a fluid saturated spongy medium is called poroelasticity (Cowin 2001). As the spongy elastic extracellular milieu of bone tissue carries living cells with both intra- and extracellular fluid so it can be viewed as a poroelastic material. Bone contains fluid in two forms, blood and the interstitial fluid. Interstitial fluid is the stage of fluid when it is contained by the pores or spaces of the solid milieu. Transportation of nutrients to bone cells immersed in milieu and carrying waste of cells away is the main functioning of bone fluid. It also helps to transfer the mineral ions to the bone tissue for reposition and salvage (Piert et al. 2001). Bone fluid is also involved in mechanosensory system of bone (Weinbaum et al. 1994). The main source of fluid flow in the bones is bone deformation, which is formed by mechanical stress on the skeleton. Such flow occurs over the cell membrane, and it is assumed that osteocytes feel the shear stress produced by the flowing fluid. Cell populations also get affected by the flow of ionic fluid due to electrical streaming potentials. Bone porousness has three levels inside cortical and cancellous bone, namely vascular porosity, lacunar-canalicular porosity, and collagen apatite porosity. All of these levels contain fluid. Osteocyte sustenance, mineral repositing and resurgence, and most likely mechanosensation all depend on the dynamics the bone fluid in the vascular porosity and lacunar-canalicular porosities. Poroelasticity and electrokinetics can be used as an efficient tool in the experimental study of local bone fluid flow, which in turn deals with quite a few questions regarding bone mineralization and mechanosensory system (Kapellos et al. 2010). 


\section{Mathematics of bone dynamics}

Utilizing the mathematical model as a link between conceptual models and experimental testing presents significant advantages. The destruction and reconstruction of bone tissue is a periodic process that involves groups of cells working together in a basic multicellular unit (BMU). Spatial movement inside the BMU is small so models based on ordinary differential equations can be used. There are delays present due to the time it takes signals to be produced and transmitted and for cells to react to them. Bone remodeling is a very complicated process and not all mechanisms are known or completely understood. There have been a number of attempts to mathematically formulate bone remodeling. It was pioneered by research group led by Hilal et al. (1964). Other investigators developed theoretical models for adaptive bone remodeling in response to altered mechanical loading (Helgason et al. 2008).

The literature provides several models that quantify aspects of bone biology and related physiology. Lemaire et al. (2004) published a quantitative cellular model describing the linking of osteoclasts and osteoblasts. This model unified several of the controlling and linking mechanisms involved in BMU concerted activities building and degrading bone. However, this model did not facilitate changes elicited through calcium homeostatic mechanisms, nor did it account for the anabolic nature of once-daily PTH administration. Mathematical models that control intracellular mechanism and described differential responses to PTH were developed in literature. A detailed overview of such methods is provided in Rao et al. (2002), Peterson and Riggs (2010).

\section{Bone dynamics and the Lattice Boltzmann method}

The Lattice Boltzmann method which is a numerical technique with intrinsic parallelism, and straightforward resolution of complex solid boundaries and multiple fluid phases has gained popularity for its ability to provide results that can be equivalent to the solution of the Navier-Stokes equations for single-fluid and multi-fluid flows through porous media (Chen and Doolen 1998).

\section{Scaffolds}

Special problems arise in the field of multidisciplinary field of tissue engineering research. In the case of damaged or lost bone one of the most promising alternative approaches to grafting is bone tissue engineering using biodegradable biomimetic scaffolds. It is expected to grow cells in a natural way i.e. similar to the process where the bone cells grow inside humans under moderate cyclic mechanical loadings.

The Lattice Boltzmann method has been extensively used for the simulation of flow conditions in combination with micro-computed tomography imaging to define the scaffold micro-architecture in a perfusion bioreactor (Martys and Chen 1996).

The scaffolds provide structural support and serve as a template for osteogenesis of newly formed tissue. Such scaffolds can be seeded with bone forming pre-osteoblastic mesenchymal stem cells (MSCs) obtained from the patient and cultured in vitro in the presence of osteogenic media. Flow-induced shear stress has been found to produce a significant stimulatory effect (Sikavitsas et al. 2003).

Recently, Lattice Boltzmann method has been used for the simulation of flow conditions in combination with micro-computed tomography imaging to define the scaffold 
micro-architecture in perfusion bio-reactor. Media perfusion bio-reactor systems have been developed to improve mass transport throughout three-dimensional tissue-engineered constructs cultured in vitro. In addition to enhancing the exchange of nutrients and wastes, these systems simultaneously deliver flow-mediated shear stresses to cells seeded within the constructs. Local shear stresses are a function of media flow rate and dynamic viscosity, bio-reactor configuration, and porous scaffold micro-architecture. Porter et al. (2005) used the Lattice Boltzmann method to simulate the flow conditions within perfused cell-seeded cylindrical scaffolds.

Perfusion bioreactors are known to exert shear stresses on cultured cells, leading to cell differentiation and enhanced extracellular matrix deposition on scaffolds. The influence of the scaffold's porous microstructure has recently been investigated by Zermatten et al. (2014) for a polycaprolactone (PCL) scaffold with a regular micro-architecture and a silk fibroin (SF) scaffold with an irregular network of interconnected pores. Mean surface shear stresses for scaffolds were calculated with the help of Lattice-Boltzmann method.

Voronov et al. (2011) used LBM and simulated solute transport in porous media using the velocity field results. Lagrangian scalar tracking (LST) method was used (to model macroscopic solute transport) in conjunction with the LBM algorithm. The fundamental hypothesis was that the solute transport behavior of passive markers is the combination of convection (obtained using the velocity field from the LBM simulations) and diffusion (obtained from a mesoscopic MonteCarlo approach that simulates Brownian motion).

\section{Volume-based fabric tensors}

The laminar and creeping fluid flows (with lower Reynolds number) can be better understood with the help of Lattice Boltzmann method.

Recently Moreno and Smedby (2014) used a novel approach to compute volume-based fabric tensors from computational fluid dynamics (CFD) simulations. Their proposed method treated a trabecular bone as a pipeline. A synthetic viscous fluid was assumed to flow from a single source located at the center of the region of interest, toward the boundaries of a spherical region of interest. In a second step the fabric tensors were computed and simulated using the Lattice-Boltzmann method.

\section{Pore-scale analysis}

In common practice, the fluid type is usually selected to be Newtonian, due to the complex and anisotropic bone geometry. Zeiser et al. (2008) simulated the flow of a Newtonian fluid on the pore-scale level of the explicit trabecular bone geometry (acquired using a microcomputed tomography technique). The Lattice Boltzmann method was used to run the simulations, a fluid was chosen to validate the Lattice Boltzmann approach for the particular area of application. It was demonstrated with useful results that LBM worked more effectively to simulate the flow in complex porous geometries as compared to other CFD approaches.

\section{LBM to demonstrate the canalicular surface dynamics}

The research group led by Kamioka calculated the effect of morphology of the canaliculi on the flow of interstitial fluid around osteocyte cell extensions (Kamioka et al. 
2012). The computational fluid dynamics analysis was made using the lattice Boltzmann method.

\section{Mathematical description of the Lattice Boltzman method}

The Lattice Boltzmann method (LBM) (Succi 2001) built on the Boltzmann equation and historically derived from the Lattice Gas Cellular Automata (LGCA) methods (Wolf-Gladrow 2000), is an emerging alternative to the Navier-Stokes (NS) based methods for the simulation of fluid flows. The motivations for its use are both theoretical and practical. On the one hand, the LBM provides a novel perspective on complex physical systems based on the averaged microscopic properties of fluids. It makes possible the expression of a wide range of macroscopic flows which are ultimately representative of similar molecular states. With its meso-scopic definition through the lattice Bhatnagar-Gross-Krook (BGK) model it also shows exact conservation properties (Bhatnagar 1954; Chen et al. 1991). On the other hand, despite its microscopic foundation, the method is easy to implement, flexible towards additional physics, and advantageous for parallelization.

The LBM is a recent method from CFD which has its roots in a highly simplified gaskinetic description, i.e. a velocity-discrete Boltzmann equation with appropriate collision term. When properly applied, the results of LBM simulations satisfy the Navier-Stokes equations in the macroscopic limit with second order of accuracy (Chen and Doolen 1998; Succi 2001).

In "Introduction" section the forms of the fluid contained inside the bone are described. The interstitial fluid is the stage of fluid when it is contained by the pores or spaces of the solid milieu. Generally, the dynamics at such scales can be classified by three types of mathematical models according to the observed scales:

1. Microscopic models at molecular scale.

2. Kinetic theories at mesoscopic scale.

3. Continuum models at macroscopic scale.

\section{Microscopic models at molecular dynamics}

In microscopic models, position and momentum of molecules can be obtained by tracking the motion of each molecule. Usually, Newton second law is used to decribe the molecular dynamics of the fluid i.e.

$$
m \mathbf{a}_{i}=\mathbf{F}_{i}
$$

where $m$ is the mass of the fluid molecule $\mathbf{a}_{i}$ is the rate of change of velocity vector of molecule $i$, and $\mathbf{F}_{i}$ is the total force and includes two parts

$$
\mathbf{F}_{i}=\mathbf{f}_{i}+\mathbf{G}_{i},
$$

where $\mathbf{f}_{i}$ is the force exerted by the fluid molecule and $\mathbf{G}_{i}$ is te external force such as gravity and electromagnetic force.

From the above equation, the location and velocity of each molecule at every time can be obtained, and then fluid density, velocity, and temperature, can be obtained from the microscopic results. In Newtonian formulation, the number of molecules is so large 
$\left(\sim 10^{23}\right)$ for a small volume of fluid in practice that it is practically impossible to describe the whole system with the most advanced computational techniques.

\section{Mesoscopic model for kinetic theory}

Mesoscopic kinetic theory is used to describe the particle distribution in compressible fluid (gas), whereas in principal kinetic theory can also be used to simulate the incompressible fluid. The basic quantity in kinetic theory is the PDF (particle distribution function) $f\left(s, v_{m}, t\right)$. It is the generalization of density $\rho$ that also consider the microscopic particle velocity. The $\rho(s, t)$ is used as the density of mass in physical space while in three-dimensional physical space and velocity space $\operatorname{PDF} f\left(s, v_{m}, t\right)$ is used as the density of mass. The pdf (particle distribution function) $f\left(s, v_{m}, t\right)$ is also related to macroscopic variables like the density and the fluid velocity $v_{f}$ from its moments. These moments are integrals of $f\left(s, v_{m}, t\right)$, weighted with some function of $v_{m}$, over the entire velocity space. For instance, the macroscopic mass density can be found as the moment

$$
\rho(s, t)=\int f\left(s, v_{m}, t\right) d^{3} v_{m}
$$

By integrating over molecular velocity space in this way, we are considering the contribution to the density of particles of all possible velocities at position $s$ and time $t$.

We can also consider the particles' contribution $v_{m} f\left(s, v_{m}, t\right)$ to the momentum density. Again considering all possible velocities, we find the macroscopic momentum density as the moment

$$
\rho(s, t) v_{f}=\int v_{m} f\left(s, v_{m}, t\right) d^{3} v_{m} .
$$

Similarly, we can find the macroscopic total energy density as the moment

$$
\rho(s, t) e(s, t)=\int\left|v_{m}\right|^{2} f\left(s, v_{m}, t\right) d^{3} v_{m},
$$

where fluid and molecular velocities can be realted with each other by the reative velocity

$$
v_{r e l}=v_{m}-v_{f}
$$

It is also considered the pdf $f\left(s, v_{m}, t\right)$ attains the equilibrium distribution $f^{e q}\left(s, v_{m}, t\right)$, when a gas has been lisolated for sufficiently long time, which is isotropic in velocity space around $v_{m}=v_{f}$ in a moving frame. Assume that the equilibrium distribution $f^{e q}\left(s,\left|v_{\text {rel }}\right|, t\right)$ is separable therefore,

$$
f^{e q}\left(s,\left|v_{r e l}\right|^{2}, t\right)=f^{e q}\left(v_{1}^{2}+v_{2}^{2}+v_{3}^{2}\right)=f_{1 D}^{e q}\left(v_{1}^{2}\right) f_{1 D}^{e q}\left(v_{1}^{2}\right) f_{1 D}^{e q}\left(v_{1}^{2}\right) .
$$

Above equation shows that the product of three $1 \mathrm{D}$ equilibrium distribution is equivalent to 3D equilibrium distribution. If the magnitude of the velocity is constant then

$$
f^{e q}\left(s,\left|v_{r e l}\right|^{2}, t\right)=\text { const }
$$

implies that

$$
\ln f^{e q}\left(v_{1}^{2}\right)+\ln f^{e q}\left(v_{2}^{2}\right)+\ln f^{e q}\left(v_{3}^{2}\right)=\mathrm{const}
$$


Above expression will be true if each 1D equilibrium distribution satisfy the following expression

$$
\ln f^{e q}\left(v_{1}^{2}\right)=a+b v_{1}^{2}
$$

which can be written as

$$
\ln f^{e q}\left(v_{1}^{2}\right)+\ln f^{e q}\left(v_{2}^{2}\right)+\ln f^{e q}\left(v_{3}^{2}\right)=3 a+b\left(v_{1}^{2}+v_{2}^{2}+v_{3}^{2}\right)=\text { const }
$$

and 3D equilibrium distribution can be written as

$$
f^{e q}\left(\left|v_{r e l}\right|\right)=e^{3 a} e^{b\left|v_{r e l}\right|^{2}}
$$

where $a$ and $b$ are constants.

Therefore the equilibrium distribution which is not unique known as the Maxwell Boltzmann distribution can be obtained as

$$
f^{e q}\left(s,\left|v_{r e l}\right|, t\right)=\left(\frac{3}{4 \pi e}\right)^{3 / 2} \rho \exp \left(-3\left|v_{r e l}\right|^{2} / 4 e\right)
$$

\section{Algorithm of LBM}

\section{Discretization}

Assume that each population $\left(f_{i}\right)$ moves with velocity $c_{i}$ from one lattice site to other in time $\Delta t$ if lattice site is uniform and regular with lattice constant $\Delta s$ and velocity components are

$$
c_{i \alpha}=n \frac{\Delta s}{\Delta t} .
$$

The population of particles at the lattice site $s$ is $f_{i}\left(s, c_{i}, t\right)$ and at the lattice site $s+c_{i} \Delta t$ is $f_{i}\left(s+c_{i} \Delta t, t+\Delta t\right)$.

\section{Reduction by method of trajectories}

From the LBE, the population of particles $f_{i}=f_{i}\left(s, c_{i}, t\right)$ satisfies the following equation

$$
\frac{\partial f_{i}}{\partial t}+\left(c_{i \alpha} \cdot \nabla\right) f_{i}=\Omega_{i}
$$

$\nabla=\frac{\partial}{\partial g_{\alpha}}, \alpha$ is space variable, where $\Omega_{i}$ (collision operator) depends upon discretized population of particles $f_{i}$ and equilibrium population of particles $f_{i}^{e q}$ which depends on the macroscopic quantities like density and velocity that are already derived through momentums of distribution function (population of particles) $f_{i}$.

The associated homogeneous equation of Eq. (14) is

$$
\frac{\partial h}{\partial t}+\vec{v} \cdot \nabla h=0, h=f_{i}, v=c_{i \alpha}
$$

which is a hyperbolic equation and have trajectories $s=s_{0}+v t$ or $s_{0}=s-v t$ where, $s_{0}$ is arbitrary and $h=h(s-v t)$ is the solution of Eq. (15). 
In the trajectory method we can write the solution of $f_{i}=f_{i}(s(\xi), t(\xi))$, where, $\xi$ is the trajectory parameter, the left hand side of $*$ can be written as a total derivative of trajectory parameter $\xi$ i.e. $\xi=t, s_{\alpha}=c_{i \alpha} \xi$.

$$
\begin{aligned}
& \frac{d f_{i}}{d \xi}=\frac{\partial f_{i}}{\partial t} \frac{\partial t}{\partial \xi}+\frac{\partial f_{i}}{\partial s_{\alpha}} \frac{d s_{\alpha}}{d \xi}=\Omega_{i}(s(\xi), t(\xi)) \\
& \frac{d f_{i}}{d \xi}=\frac{\partial f_{i}}{\partial t}+c_{i \alpha} \frac{\partial f_{i}}{\partial s_{\alpha}}
\end{aligned}
$$

thus

$$
\frac{d f_{i}}{d \xi}=\Omega_{i}(s(\xi), t(\xi))
$$

Choosing the initial point $\left(s_{0}, t_{0}\right)$ i.e. $\xi=0$

we can now integrate on $[0, \Delta t]$

$$
\begin{aligned}
& \int_{0}^{\Delta t} \frac{d f_{i}}{d \xi} d \xi=\int_{0}^{\Delta t} \Omega_{i}(s(\xi), t(\xi)) d \xi \\
& f_{i}\left(s_{0}+c_{i} \Delta t, t_{0}+\Delta t\right)-f_{i}\left(s_{0}, t_{0}\right)=\int_{0}^{\Delta t} \Omega_{i}\left(s_{0}+c_{i} \xi, t_{0}+\xi\right) d \xi .
\end{aligned}
$$

Since, $\left(s_{0}, t_{0}\right)$ is the arbitrary point, therefore

$$
f_{i}\left(s+c_{i} \Delta t, t+\Delta t\right)-f_{i}(s, t)=\int_{0}^{\Delta t} \Omega_{i}\left(s+c_{i} \xi, t+\xi\right) d \xi
$$

\section{Space-time integration method}

To approximate the RHS of Eq. (21), RK and Crank Nicolson schemes can be used to integrate the collision operation via rectangular cube. The first order discretization approximate the collision operator integral by following expression:

$$
f_{i}\left(s+c_{i} \Delta t, t+\Delta t\right)-f_{i}(s, t)=\Delta t \Omega_{i}(s, t)
$$

or

$$
f_{i}\left(s+c_{i} \Delta t, t+\Delta t\right)=f_{i}(s, t)+\Delta t \Omega_{i}(s, t)
$$

Equation (23) is called is called the Lattice Boltzman Equation (LBE) and is used for LB simulations, it can't be solved unless the collision operator $\Omega_{i}(s, t)$ is defined. For this purpose, we use collision operator that is widely used for LBE.

\section{BGK collision operator}

To approximate the collision operator $\Omega_{i}$ defined $\Omega_{i}$ in terms of known variable i.e. distribution function $f_{i}$ (population of particles) and equilibrium distribution function $f_{i}^{e q}$ (population of particles at equilibrium condition). 
Assume that $\Omega_{i} \propto f_{i}-f_{i}^{e q}$

$$
\begin{aligned}
& \Omega_{i} c_{i} \propto f_{i} c_{i}-f_{i}^{e q} c_{i} \\
& \sum_{i} \Omega_{i} c_{i} \propto \sum_{i}\left(f_{i} c_{i}-f_{i}^{e q} c_{i}\right)
\end{aligned}
$$

The Navier-Stokes behavior requires that mass and momentum is conserved, therefore

$$
\sum_{i} \Omega_{i} c_{i}=\sum_{i}\left(f_{i} c_{i}-f_{i}^{e q} c_{i}\right)=0
$$

from the BGK collision operator, we obtain

$$
\Omega_{i}=\frac{-f_{i}-f_{i}^{e q}}{\lambda}
$$

which shows that trend of population of particle $f_{i}$ to equilibrium population of $f_{i}^{e q}$ in time $\lambda$, which is also called the relaxation time. Thus the lattice Boltzmann equation with BGK collision operator is given by the expression.

$$
f_{i}\left(s+c_{i} \Delta t, t+\Delta t\right)=f_{i}(s, t)-\frac{\Delta t}{\lambda}\left(f_{i}(s, t)-f_{i}^{e q}(s, t)\right)
$$

or

$$
f_{i}(t+\Delta t)=\left(1-\frac{\Delta t}{\lambda}\right) f_{i}+\frac{\Delta t}{\lambda} f_{i}^{e q}
$$

In the literature Wang et al. (2016), Hübner (2010) it has been verified the LB with BGK operator can reproduce the NSE and continuity equation.

\section{Lattice Boltzmann computational designs}

Different models and designs exist in the literature for ' $X$ ' dimensions and ' $Y$ ' discrete velocities of the Lattice Boltzmann method. Such models are characterized as DXQY models. A list of some commonly used models is provided in Table 1. The basic flow chat for the algorithm is presented in Fig. 1. It is obvious from these steps that a complete description is desired of the technique for the biological problems in general and for the bone remodelling and other applications in the field of osteology, in particular. We have explained in detail the analytic and numerical approaches for the Lattice Boltzmann method in second part of this article.

\section{Conclusions}

In this article, we have outlined the deep mechanism of a continually bone renewal practice, called bone remodeling. The mathematical models of bone remodeling present in literature, concentrate on different aspects. Some approaches were concerned with bone mechanics and its effects on bone formation and resorption, while other modelling efforts were based on the signaling pathways among bone forming and resorbing cells or described the behavior of both cells in a microenvironment section called a basic multicellular unit (BMU). A few efforts on mathematical modeling of bone remodeling have been discussed in "Bone" section. 
Table 1 LBM models and their respective application

\begin{tabular}{lll}
\hline Model & References & \\
\hline D2Q7 & Zhang et al. (2004) & $\begin{array}{c}\text { To study solid-fluid interactions and free energy for fluid } \\
\text { systems } \\
\text { Thermal conductivity for microscale random porous } \\
\text { media }\end{array}$ \\
D2Q9 & Wang et al. (2007) & $\begin{array}{l}\text { To study two-phase flows on partial wetting surface } \\
\text { The effect of biofilm permeability on bio-clogging of } \\
\text { porous media lid-driven cavity }\end{array}$ \\
D3Q19 & Pintelon et al. (2012), Contreras (2013) & $\begin{array}{c}\text { Microscale fluid flow analysis in a human osteocyte cana- } \\
\text { liculus particle-based biological system simulator }\end{array}$ \\
D3Q27 & Boulianne et al. (2008), Kamioka et al. (2012) & (2007)
\end{tabular}

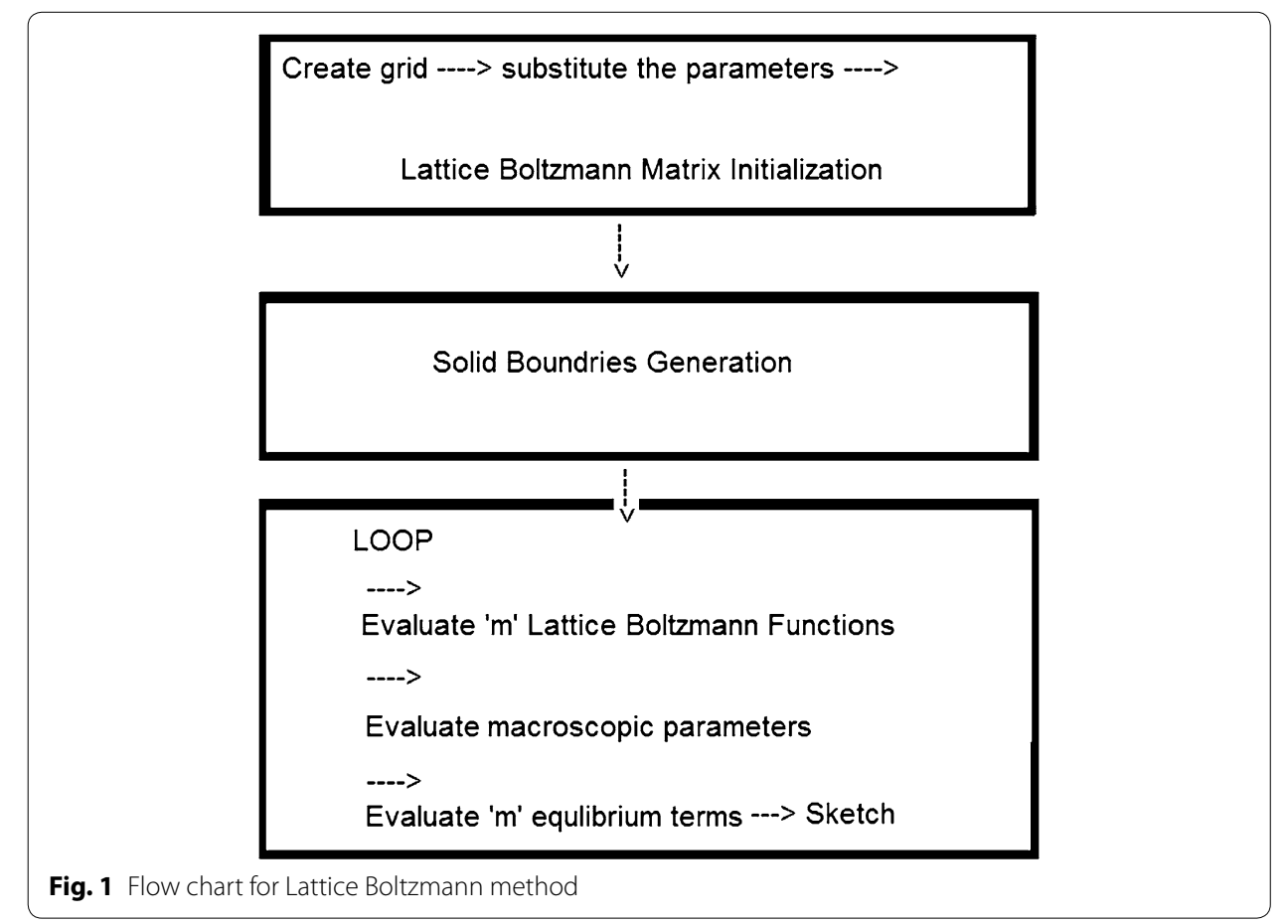

Lately, Lattice Boltzmann method (LBM) has been considered for the simulation of flow conditions in combination with micro-computed tomography imaging to describe the scaffold micro-architecture in perfusion bio-reactor. Media perfusion bio-reactor systems have been developed to improve mass transport throughout three-dimensional tissue-engineered constructs cultured in vitro. In addition to enhancing the exchange of nutrients and wastes, these systems simultaneously deliver flow-mediated shear stresses to cells seeded within the constructs. Local shear stresses are a function of media flow rate and dynamic viscosity, bio-reactor configuration, and porous scaffold micro-architecture. The benefit of the Lattice Boltzmann is that it can be coded for parallel machines and GPUs directly. Thus, executions for treating with complex hyperbolic PDEs, like the Navier-Stokes PDE, can be found proficiently. This work will help the reader to apply the most appropriate multiscale modelling strategy and will help to understand the deep mechanism of bone remodeling, since the study is complex and this study will help to 
model the the influence of the microscopic features such as the surface roughness of the canalicular wall on the profiles of mechanical loading-induced flow of interstitial fluid. In future, we will provide some useful numerical tools to run such simulations.

\section{Authors' contributions}

SJ conceived the manuscript. AS collected materials and methods. KM did the analytic modelling. AS, SIB and QAC did numerical modelling. All the authors did the literature review. All authors read and approved the final manuscript.

\section{Author details}

${ }^{1}$ Department of Mathematics, Comsats Institute of Information Technology, Lahore 54000, Pakistan. ${ }^{2}$ Department of Mathematics \& Statistics, FBAS, IIUI, Islamabad 44000, Pakistan. ${ }^{3}$ Department of Mathematics, University of Engineering and Technology, Lahore 54890, Pakistan.

\section{Competing interests}

The authors declare that they have no competing interests.

Availability of data and materials

Not applicable.

\section{Funding}

Not applicable.

\section{Publisher's Note}

Springer Nature remains neutral with regard to jurisdictional claims in published maps and institutional affiliations.

Received: 9 October 2017 Accepted: 30 October 2017

Published online: 13 November 2017

\section{References}

Aidun CK, Clausen JR (2010) Lattice-Boltzmann method for complex flows. Ann Rev Fluid Mech 42:439-472

Alemani D, Pappalardo F, Pennisi M, Motta S, Brusic V (2012) Combining cellular automata and lattice boltzmann method to model multiscale avascular tumor growth coupled with nutrient diffusion and immune competition. J Immunol Methods 376(1):55-68

Bhatnagar PL, Gross EP, Krook M (1954) A model for collision processes in gases. I. Small amplitude processes in charged and neutral one-component systems. Phys Rev 94(3):511

Boulianne L, Al Assaad S, Dumontier M, Gross WJ (2008) Gridcell: a stochastic particle-based biological system simulator. BMC Syst Biol 2(1):66

Canalis E, McCarthy T, Centrella M (1988) Growth factors and the regulation of bone remodeling. J Clin Invest 81(2):277

Chen S, Doolen GD (1998) Lattice Boltzmann method for fluid flows. Ann Rev Fluid Mech 30(1):329-364

Chen I, Downing R, Keshock EG, Al-Sharif M (1991) Measurements and correlation of two-phase pressure drop under microgravity conditions. J Thermophys Heat Transfer 5(4):514-523

Contreras JO (2013) Efficient algorithms for the realistic simulation of fluids

Cowin SC (2001) Mechanosensory mechanisms in bone. Lecture notes-ABIOMED. Centre for Excellence for Applied Biomedical Modelling and Diagnostics, Warsaw

Hall DJ, Urban RM, Pourzal R, Turner TM, Skipor AK, Jacobs JJ (2017) Nanoscale surface modification by anodic oxidation increased bone ingrowth and reduced fibrous tissue in the porous coating of titanium-alloy femoral hip arthroplasty implants. J Biomed Mater Res 105(2):283-290

Helgason B, Perilli E, Schileo E, Taddei F, Brynjólfsson S, Viceconti M (2008) Mathematical relationships between bone density and mechanical properties: a literature review. Clin Biomech 23(2):135-146

Hilal SK, Mosser DG, Loken MK, Johnson RW (1964) A new technique for high-resolution density gradient-separation of bone-marrow cells. Ann NY Acad Sci 114(1):661-676

Hübner TH (2010) A monolithic, off-lattice approach to the discrete Boltzmann equation with fast and accurate numerical methods. PhD thesis, PhD Thesis, TU Dortmund

Kamioka H, Kameo Y, Imai Y, Bakker AD, Bacabac RG, Yamada N, Takaoka A, Yamashiro T, Adachi T, Klein-Nulend J (2012) Microscale fluid flow analysis in a human osteocyte canaliculus using a realistic high-resolution image-based threedimensional model. Integr Biol 4(10):1198-1206

Kapellos GE, Alexiou TS, Payatakes AC (2010) Theoretical modeling of fluid flow in cellular biological media: an overview. Math Biosci 225(2):83-93

Kerschnitzki M, Kollmannsberger P, Burghammer M, Duda GN, Weinkamer R, Wagermaier W, Fratzl P (2013) Architecture of the osteocyte network correlates with bone material quality. J Bone Miner Res 28(8):1837-1845

Lemaire V, Tobin FL, Greller LD, Cho CR, Suva LJ (2004) Modeling the interactions between osteoblast and osteoclast activities in bone remodeling. J Theor Biol 229(3):293-309

Martys NS, Chen H (1996) Simulation of multicomponent fluids in complex three-dimensional geometries by the Lattice Boltzmann method. Phys Rev E 53(1):743

Mohamad AA (2011) Lattice Boltzmann method: fundamentals and engineering applications with computer codes. Springer Science \& Business Media, Berlin 
Moreno R, Smedby Ö (2014) Volume-based fabric tensors through Lattice-Boltzmann simulations. In: 22nd international conference on pattern recognition (ICPR). IEEE, New York. pp 3179-3184

Mullender M, Huiskes R (1997) Osteocytes and bone lining cells: which are the best candidates for mechano-sensors in cancellous bone? Bone 20(6):527-532

Parfitt A (1987) Bone remodeling and bone loss: understanding the pathophysiology of osteoporosis. Clin Obstet Gynecol 30(4):789-811

Peterson MC, Riggs MM (2010) A physiologically based mathematical model of integrated calcium homeostasis and bone remodeling. Bone 46(1):49-63

Piert M, Zittel TT, Becker GA, Jahn M, Stahlschmidt A, Maier G, Machulla H-J, Bares R (2001) Assessment of porcine bone metabolism by dynamic [18f] fluoride ion pet: correlation with bone histomorphometry. J Nucl Med 42(7):1091-1100

Pintelon TR, Picioreanu C, van Loosdrecht M, Johns ML (2012) The effect of biofilm permeability on bio-clogging of porous media. Biotechnol Bioeng 109(4):1031-1042

Porter B, Zauel R, Stockman H, Guldberg R, Fyhrie D (2005) 3-d computational modeling of media flow through scaffolds in a perfusion bioreactor. J Biomech 38(3):543-549

Rao CV, Wolf DM, Arkin AP (2002) Control, exploitation and tolerance of intracellular noise. Nature 420(6912):231

Sikavitsas VI, Bancroft GN, Holtorf HL, Jansen JA, Mikos AG (2003) Mineralized matrix deposition by marrow stromal osteoblasts in 3d perfusion culture increases with increasing fluid shear forces. Proc Natl Acad Sci 100(25):14683-14688

Succi S (2001) The Lattice Boltzmann equation: for fluid dynamics and beyond. Oxford, Oxford university press

Voronov RS, VanGordon SB, Sikavitsas VI, Papavassiliou DV (2011) Efficient lagrangian scalar tracking method for reactive local mass transport simulation through porous media. Int J Num Methods Fluids 67(4):501-517

Wang M, Wang J, Pan N, Chen S (2007) Mesoscopic predictions of the effective thermal conductivity for microscale random porous media. Phys Rev E 75(3):036702

Wang J, Chen L, Kang Q, Rahman SS (2016) The Lattice Boltzmann method for isothermal micro-gaseous flow and its application in shale gas flow: a review. Int J Heat Mass Transf 95:94-108

Weinbaum S, Cowin SC, Zeng Y (1994) A model for the excitation of osteocytes by mechanical loading-induced bone fluid shear stresses. J Biomech 27(3):339-360

Wolf-Gladrow DA (2002) 1. Introduction. In: Lattice gas cellular automata and Lattice Boltzmann models. Springer, Berlin. pp 1-13

Yan Y, Zu Y (2007) A lattice boltzmann method for incompressible two-phase flows on partial wetting surface with large density ratio. J Comput Phys 227(1):763-775

Zeiser T, Bashoor-Zadeh M, Darabi A, Baroud G (2008) Pore-scale analysis of newtonian flow in the explicit geometry of vertebral trabecular bones using lattice boltzmann simulation. Proc Inst Mech Eng 222(2):185-194

Zermatten E, Vetsch JR, Ruffoni D, Hofmann S, Müller R, Steinfeld A (2014) Micro-computed tomography based computational fluid dynamics for the determination of shear stresses in scaffolds within a perfusion bioreactor. Ann Biomed Eng 42(5):1085-1094

Zhang J, Li B, Kwok DY (2004) Mean-field free-energy approach to the Lattice Boltzmann method for liquid-vapor and solid-fluid interfaces. Phys Rev E 69(3):032602

\section{Submit your manuscript to a SpringerOpen ${ }^{\circ}$ journal and benefit from:}

- Convenient online submission

Rigorous peer review

- Open access: articles freely available online

- High visibility within the field

Retaining the copyright to your article

Submit your next manuscript at $\gg$ springeropen.com 